\title{
HEMATOPOIESIS
}

\section{Commitment to the Monocytic Lineage Occurs in the Absence of the Transcription Factor PU.1}

\author{
By Gregory W. Henkel, Scott R. McKercher, Pieter J .M. Leenen, and Richard A. Maki
}

\begin{abstract}
Mice homozygous for the disruption of the PU.1 (Spi-1) gene do not produce mature macrophages. In determining the role of PU.1 in macrophage differentiation, the present study investigated whether or not there was commitment to the monocytic lineage in the absence of PU.1. Early PU.1-/myeloid colonies were generated from neonate liver under conditions that promote primarily macrophage and granulocyte/macrophage colonies. These PU.1-/- colonies were found to contain cells with monocytic characteristics as determined by nonspecific esterase stain and the use of monoclonal antibodies that recognize early monocyte precursors, including Moma-2, ER-MP12, ER-MP20, and ER-MP58.
\end{abstract}

D IFFERENT DEVELOPMENTAL stages, from progenitors to mature cells, have been defined during the process of monocyte/macrophage differentiation. Nevertheless, the critical molecular switches necessary to orchestrate monocyte/macrophage differentiation are poorly understood. One important regulator required for monocyte/macrophage development is the transcription factor PU.1 (Spi-1). In PU.1-/- mice, mature $\mathrm{F} 4 / 80$ positive macrophages are not observed in various tissues known to contain these cells. ${ }^{1-3}$ The use of in vitro colonyforming assays further showed that no distinctive macrophage colonies are generated from yolk sac, fetal liver, neonate liver, or bone marrow cells from PU.1 null animals. ${ }^{4-7}$ Likewise, PU.1-/- embryonic stem cells, when induced to differentiate along the macrophage lineage, do not produce mature F4/80 positive cells. ${ }^{4,8}$ It is not clear how PU. 1 is involved in the regulation of macrophage development. The defect in macrophage differentiation may occur before commitment, or it may occur after commitment at an early point in monocyte maturation. Therefore, determining where the block in monocyte/ macrophage development occurs is an important step in discovering the function of PU.1 in the differentiation of this lineage.

Although mature macrophages are not detected in PU.1-/animals, it has never been shown if early monocytic precursors exist in PU.1-/- mice. The earliest cell described in the monocytic lineage is the monoblast, which on division gives rise to the promonocyte. ${ }^{9}$ Promonocytes are the direct precursors to monocytes. Low F4/80 expression is detectable as early as the promonocyte stage and increases as the cells mature. ${ }^{9-11}$

Other surface markers found on early macrophage precursors include receptors for immunoglobulin $\mathrm{G}(\mathrm{IgG})$ and complement. ${ }^{12}$ Characteristic enzyme activity associated with the monocytic lineage, such as nonspecific esterase and lysozyme, can be detected as early as the monoblast stage of development. ${ }^{13}$ Monoblasts and promonocytes also show moderate peroxidase activity that diminishes as the cells mature. ${ }^{13}$ These early macrophage precursors also engage in pinocytosis and phagocytosis, although less actively relative to mature macrophages. ${ }^{13}$

Recently, several monoclonal antibodies, including ERMP12, ER-MP20, and ER-MP58, have been shown to recognize antigens associated with early precursor cells of the monocyte/macrophage lineage. ${ }^{14,15}$ ER-MP12 ${ }^{\text {hi}} /$ ER-MP20- ${ }^{-}$ER-
In addition, early myeloid cells could be grown from PU.1-/fetal liver cultures in the presence of granulocyte-macrophage colony-stimulating factor (GM-CSF). Similar to the PU.1 null colonies, the GM-CSF-dependent cells also possessed early monocytic characteristics, including the ability to phagocytize latex beads. The ability of PU.1-/ - progenitors to commit to the monocytic lineage was also verified in vivo by flow cytometry and cytochemical analysis of primary neonate liver cells. The combined data shows that PU.1 is absolutely required for macrophage development after commitment to this lineage.

(C) 1999 by The American Society of Hematology.

$\mathrm{MP}^{2} 2^{+} / \mathrm{ER}-\mathrm{MP} 20^{+}$, and ER-MP12-/ER-MP20 ${ }^{\text {hi }}$ population of cells in bone marrow contain macrophage colony or clusterforming progenitors. ${ }^{16}$ In addition, these three populations represent distinct stages of increasing monocyte maturation as cells develop from ER-MP12 ${ }^{\text {hi }}$ only to ER-MP20 hi. Mature macrophages no longer express ER-MP12 or ER-MP20. ${ }^{16}$ High ER-MP58 expression is found on the majority of M-CSFresponsive monocyte/macrophage precursors in the bone marrow, but diminishes as the cells mature toward macrophages. ${ }^{15,17}$

Another monoclonal antibody, MOMA-2, recognizes both mature subsets of macrophages and bone marrow monocyte/ macrophage precursors. ${ }^{18}$ The antigen recognized by this antibody is primarily intracellular, although some level of surface expression has been shown. Moma-2 is reported not to react with neutrophils and lymphocytes. ${ }^{18}$ In contrast, various levels of ER-MP58 expression can be found on the majority of cells in normal bone marrow. ${ }^{14}$ Similarly, both ER-MP12 and ERMP20 recognize other hematopoietic cell types, including lymphocytes and granulocytes. ${ }^{16,19}$ ER-MP12 has also been shown to be a marker on multiprogenitor cells and costains with other stem cell markers, including Sca-1 and c-Kit. ${ }^{20}$ The ER-MP12 antibody has recently been found to recognize the CD31 antigen (PECAM-1), whereas ER-MP20 recognizes the Ly-6C antigen. ${ }^{21,22}$ Although ER-MP12, ER-MP20, and ERMP58 recognize other cells, the use of these antibodies together with Moma-2 should be valuable for identifying early monocytes.

From The Burnham Institute, La Jolla, CA, and Department of Immunology, Erasmus University, Rotterdam, Netherlands.

Submitted September 14, 1998; accepted December 11, 1998.

G.W.H. and S.R.M. contributed equally to this study.

Supported by National Institutes of Health Grant No. AI30656.

Address reprint requests to Richard A. Maki, PhD, The Burnham Institute, 10901 N Torrey Pines Rd, La Jolla, CA 92037.

The publication costs of this article were defrayed in part by page charge payment. This article must therefore be hereby marked "advertisement" in accordance with 18 U.S.C. section 1734 solely to indicate this fact.

(C) 1999 by The American Society of Hematology.

0006-4971/99/9309-0015\$3.00/0 
In this report, we demonstrate using monoclonal antibodies, Moma-2, ER-MP12, ER-MP20, and ER-MP58, along with cytochemical and functional assays, that PU.1-/- hematopoietic progenitors commit to the monocytic lineage, but are blocked in their ability to become mature macrophages.

\section{MATERIALS AND METHODS}

Mice. $\mathrm{C} 57 \mathrm{BL} / 6 \times 129 \mathrm{~Sv}$ PU. 1 targeted mice were generated as previously reported. ${ }^{2}$ PU.1 null fetal and neonatal mice were produced by mating PU.1 targeted heterozygous or PU.1 null, normal bone marrow-reconstituted adults. Genotyping of pups was accomplished by genomic DNA polymerase chain reaction (PCR). ${ }^{2}$

Antibodies. Monoclonal antibodies: ER-MP12 (MP12), ER-MP20 (MP20), and ER-MP58 (MP58) ${ }^{14}$; Moma-2 (Harlan Bioproducts, Indianapolis, IN); F4/80 (C1:A3-1; Biosource Int'1, Camarillo, CA); CD11b, CD18, CD16/32, c-Kit, Sca-1, Gr-1 (PharMingen, San Diego, CA). Secondary antibodies: anti-Rat Ig2a fluorescein isothiocyanate (FITC) and anti-Rat Ig2a Biotin (PharMingen); and Goat $\left(\mathrm{Fab}^{\prime}\right)_{2}$ anti-Rat IgG-PE (Biosource Int'1). Polyclonal antibodies: Rabbit antigranulocyte-macrophage colony-stimulating factor (GM-CSF) receptor $\alpha$ and control Rabbit IgG (Santa Cruz Biotechnology, Santa Cruz, CA).

Colony assays. Normal and PU.1 null livers were removed from neonatal mice and passaged through a nylon mesh screen followed by a 25 -gauge needle. Red blood cells were lysed with a $0.15 \mathrm{~mol} / \mathrm{L}$ solution of ammonium chloride. Cells were plated in $1 \%$ methylcellulose containing media with $15 \%$ heat inactivated fetal bovine serum (FBS), $50 \mathrm{ng} / \mathrm{mL}$ stem cell factor (SCF; R\&D Systems, Minneapolis, MN), 2 $\mathrm{ng} / \mathrm{mL}$ recombinant GM-CSF (R\&D Systems), $100 \mathrm{U} / \mathrm{mL}$ recombinant interleukin-3 (IL-3), and 5,000 U/mL human recombinant macrophage colony-stimulating factor (M-CSF). (Both the IL-3 and M-CSF were kindly provided by David Hume, Queensland University, Australia.) Colonies were scored after 7 days in culture.

Enzyme histochemistry. Cells for enzyme histochemical analysis were spun onto slides with a cytocentrifuge and fixed in a mixture of Citrate Acid Solution (Sigma, St Louis, MO), acetone, and formaldehyde (CAF). To identify nonspecific esterase (NSE) activity, a commercially available kit for NSE staining (Sigma) was used. The NSE substrate in this kit was $\alpha$-naphthyl acetate. 3-amino-9 ethylcarbazole was used as a substrate for peroxidase staining. ${ }^{23}$

Immunohistochemical stains. Slides were fixed in CAF and incubated with primary antibodies. To visualize cells recognized by the antibodies, immunoperoxidase staining was performed with the Vectastain Elite ABC and Vector VIP substrate kit (Vector Labs, Burlingame, CA). Cells were counterstained with Vector Methyl Green (Vector Labs).

Reverse transcriptase-PCR analysis. $\quad$ RNA from normal and PU.1 null colonies was isolated using Trizol according to the manufacturer's protocol (GIBCO/BRL, Gaithersburg, MD). Two micrograms of total RNA was used to generate single strand cDNA. PCR conditions were performed as previously described, except the magnesium concentration was increased to $3 \mathrm{mmol} / \mathrm{L}$ final concentration. ${ }^{8}$ The PCR primers for PU.1 and GM-CSF receptor $\alpha$ have been reported. ${ }^{8}$ The primers for these genes amplify across introns to control for genomic contamination.

Fetal liver cultures. A single cell suspension of normal or PU.1 null E17-E18 fetal liver cells was plated in T25 flasks in Iscove's media with $20 \%$ FBS and $2.5 \mathrm{ng} / \mathrm{mL}$ GM-CSF. PU.1-/- myeloid cells growing from these cultures were established away from the fetal liver stroma and sustained as a suspension cell culture with $1.25 \mathrm{ng} / \mathrm{mL}$ GM-CSF. Clones were generated by limiting dilution in 96 well plates.

Phagocytic assay. GM-dependent PU.1-1- cells were plated in media with $1.25 \mathrm{ng} / \mathrm{mL}$ GM-CSF and $3.6 \times 10^{9}$ particles $/ \mathrm{mL}$ of $0.2 \mu \mathrm{m}$ carboxylated fluorescent latex beads (Molecular Probe, Eugene OR). After 1 or 4 hours, cells were washed with fluorescence-activated cell sorter (FACS) buffer (phosphate-buffered saline [PBS] with 2\% FBS and $0.1 \%$ azide), resuspended in PBS, layered over FBS, and pelleted through the serum. Cells were fixed in $0.8 \%$ paraformaldehyde for 5 minutes on ice, washed, and resuspended in FACS buffer for analysis. In addition, slides were made for microscopic examination.

Flow cytometry. After a single cell suspension of neonate or fetal liver was prepared, the cells were incubated with DNase I at $100 \mathrm{U} / \mathrm{mL}$ final concentration for 30 minutes at $37^{\circ} \mathrm{C}$. Cells were washed in FACS buffer and prestained in $50 \mu \mathrm{L}$ of FACS buffer with Fc Block (PharMingen) (CD 16/32) for 15 minutes before the addition of the appropriate monoclonal antibodies. Cell surface fluorescence was determined using FITC and r-phycoerythrin (PE) directly conjugated antibodies. For unconjugated primary antibodies, a biotinylated, FITCor PE-conjugated secondary antibody was used. A 1:500 dilution of Streptavidin-Cy-Chrome (PharMingen) was used in the triple staining experiments. Appropriate isotype controls were also included. Events were collected from samples using a Becton Dickinson FACScan (Becton Dickinson, San Jose, CA), and analysis was performed using Cell Quest (Becton Dickinson).

Cell sorting. Normal and PU.1 null liver cells were incubated with MP12, followed by anti-Ig2a-biotin and Streptavidin-PE (PharMingen). Moma-2 FITC was added next, followed by anti-FITC Microbeads (Miltenyi Biotec, Auburn, CA). The cells were washed, resuspended in FACS buffer, and run over a steel wool column surrounded by a magnet (Miltenyi Biotec). The nonmagnetic fraction was collected into one tube. The cells retained in the steel wool were recovered by removing the magnet and washing the cells with FACS buffer. The enrichment of the magnetic fraction of ER-MP12 ${ }^{+}$Moma- $2^{+}$cells was verified by flow cytometry.

\section{RESULTS}

Cells from PU.1 null myeloid colonies show features of monocytic precursors. It has recently been reported by our laboratory and others that neutrophil colonies can be generated from progenitors isolated from PU.1-/- neonatal and fetal liver. ${ }^{6,7}$ The production of neutrophil colonies is consistent with the presence of neutrophils detected in PU.1-/- mice. ${ }^{2}$ The identification of granulocyte colony-forming cells (G-CFC) from PU.1-/- liver suggests that either the earlier granulocyte/ macrophage (GM) progenitor also exists or some alternative pathway bypassing the GM stage of development results in the production of neutrophil colonies. If GM progenitors exist in the PU.1-/- mice, then there may also be commitment down the monocytic lineage. To determine if PU.1-/- progenitors can commit to the monocytic lineage, colony-forming (CFU) assays were set up with cells from normal and PU.1 null neonate liver under myeloid growth factor conditions that included SCF, IL-3, GM-CSF, and M-CSF.

These growth factor conditions were found to induce primarily macrophage and GM colonies from normal progenitors (Table 1). In addition, the GM colonies contained a higher proportion of macrophages than neutrophils. No erythroid colonies were detected on the CFU plates. Cells isolated from PU.1 null neonate liver also formed colonies under these same conditions. In agreement with our previously published results, the number of PU.1-/- CFU cells from neonate liver (Table 1) was significantly reduced compared with normal livers. ${ }^{6}$ Individual PU.1-/- colonies were isolated and stained by WrightGiemsa. In some cases, neutrophil-only colonies were identified; however, most of the colonies examined contained immature mononuclear cells or a mixture of mononuclear cells and some neutrophils (Fig 1A). No erythroid or typical macrophage colonies were detected on the PU.1-/- CFU plates. Also shown in the same 
Table 1. Colony-Forming Assays With Cells From Normal or PU.1 Null Neonate Livers

\begin{tabular}{|c|c|c|c|c|}
\hline Normal & M & GM & $\mathrm{N}$ & $\begin{array}{l}\text { Colonies/ } \\
\text { 7,500 Cells }\end{array}$ \\
\hline Exp. 1 & $12.3 \pm 2.1$ & $3.3 \pm 0.6$ & $6.3 \pm 1.3$ & $21.7 \pm 3.5$ \\
\hline Exp. 2 & $21.7 \pm 7.6$ & $7.0 \pm 1.7$ & $4.7 \pm 2.3$ & $33.3 \pm 11.0$ \\
\hline PU.1-I- & \multicolumn{4}{|c|}{ Colonies/7,500 Cells* } \\
\hline Exp. 1 & \multicolumn{2}{|c|}{$6.4 \pm 1.4$} & & \\
\hline Exp. 2 & \multicolumn{2}{|c|}{$2.4 \pm 0.5$} & & \\
\hline
\end{tabular}

Seven thousand five hundred normal and 1-1.5 × 105 PU.1-/neonate liver cells were plated in triplicate. Normal colonies were scored for macrophage (M), both macrophages and neutrophils (GM), and neutrophil $(\mathrm{N})$ colonies. All values represent the means of triplicate determination $+/$ - standard deviation.

*The PU.1 null CFU plates contained only myeloid colonies.

figure, a normal macrophage colony stained with Wright-Giemsa (Sigma) illustrates the morphology of both mature and immature macrophages. The PU.1 null mononuclear cells showed a similar morphology to that of normal early macrophage precursors, including in some cases, the presence of vacuoles. However, early granulocytic cells also have a similar morphology.

To determine if these early PU.1 null myeloid cells were monocytic, normal and PU.1-/- colonies were isolated and stained for NSE activity. NSE-positive normal macrophages, as indicated by the dark stain, are shown in Fig 1B. Similarly, some of the early PU.1-/- myeloid cells also showed NSE activity. Neutrophils from normal and PU.1 null CFU plates were negative for NSE activity. This is in agreement with what has been reported, that granulocyte esterase activity can be distinguished from esterase activity found in cells of the monocyte lineage. ${ }^{24,25}$ However, weak activity in granulocytes has been observed when the $\mathrm{pH}$ in the NSE reaction goes above 7.0 and the incubation time is prolonged. ${ }^{25}$ Although this was not a problem with the conditions used in this study, we decided to verify that the NSE-positive reaction was monocyte-specific. Sodium fluoride (NaF) specifically inhibits NSE activity found in cells of the monocytic lineage, whereas the esterase activity in granulocytes is $\mathrm{NaF}$ resistant. ${ }^{25}$ The addition of $\mathrm{NaF}$ to the NSE reaction inhibited the appearance of dark staining cells on both normal and PU.1-/- slides, confirming that the esterase activity measured in the PU.1-/- cells was monocyte derived (data not shown).

The PU.1-/- cells in these colonies clearly represent an early stage in myeloid development. Many early and late myeloid markers are shared by monocytic and granulocytic cells. Because Moma-2 recognizes cells of the monocyte lineage, we used this monoclonal antibody to stain pooled myeloid colonies from normal or PU.1-/- CFU plates. Normal macrophages and early precursors were positive for Moma-2, whereas neutrophils were weakly stained or completely Moma-2 negative (Fig 1C). Most of the PU.1 null early myeloid cells were also Moma-2 positive. However, between $10 \%$ and $20 \%$ of the cells were either weakly positive or did not stain with Moma-2. Additional monoclonal antibodies recognizing antigens found on monocytic precursors, including ERMP12, ER-MP20, and ER-MP58, were used to stain cytospins of cells pooled from PU.1-/- myeloid colonies. As shown in Table 2, the majority of the cells were ER-MP12- and
ER-MP58-positive, whereas $30 \%$ to $50 \%$ of the cells were ER-MP20-positive. The presence of these markers on the majority of the cells from the PU.1-/- colonies shows that these cells are blocked at a very early stage in development. This is further shown by the detection of c-Kit on most of these cells. We also stained the cells with an antibody that recognizes the Gr-1 (Ly-6G) antigen found on early and late granulocytes. ${ }^{26}$ In addition, Gr-1 is expressed transiently on cells of the monocytic lineage. ${ }^{26}$ Ten to twenty percent of the PU.1-/cells were Gr-1 positive. Less than $50 \%$ of the Gr-1-positive cells were strongly positive for this antigen, whereas the majority of the cells were weakly positive. High levels of Gr-1 expression are reported to be found on neutrophils, whereas low to moderate levels of Gr-1 are found on myeloblasts and cells of the monocytic lineage. ${ }^{26}$ In agreement with what has been reported, there were no PU.1-/- cells expressing the late monocytic F4/80 marker, although F4/80-positive cells were detected on slides of normal cells. In addition, except for a rare one or two weakly positive cells, CD11b protein could not be detected in the PU.1-/- colony-derived myeloid cells, but was readily detected on normal myeloid cells.

The presence of cells in PU.1 null colonies with early monocyte morphology, NSE activity, and early precursor antigens, including Moma-2, supports the hypothesis that PU.1-/progenitors commit to the monocytic lineage.

PU.1-/- monocytic precursors can be expanded from fetal liver with GM-CSF. We have reported minimal levels of GM-CSF receptor (GMR) on PU.1-/- cells, either from short-term neonate liver cultures or freshly isolated cells from bone marrow and liver of 9-day-old PU.1 null animals. ${ }^{6}$ In contrast, no c-fms positive cells were detected in that study. GM-CSF has been shown to be important for sustaining multipotent progenitors as well as regulating myeloid development. ${ }^{27}$ Therefore, we stained slides of normal and PU.1 null colonies with an antibody to GMR and found greater than 50\% of normal and PU.1 null cells were positive for GMR (Fig 2A). Control antibody did not stain either normal or PU.1-/- cells. As further evidence for specificity, we observed that anti-GMR did not stain an IL-7-dependent B-cell line generated from normal C57BL-6 mice (data not shown). RNA was isolated from both normal and PU.1 null myeloid cells and examined for GMR- $\alpha$ gene expression by reverse transcriptase-PCR (RTPCR). GMR- $\alpha$ message was detected in both normal and PU.1 null samples (Fig 2B). To verify that normal cells had not

Table 2. Antibody Staining of PU.1-/- Myeloid Colonies

\begin{tabular}{lc}
\hline Markers & Percent Positive* \\
\hline ER-MP12 & $>90 \%$ \\
ER-MP58 & $>90 \%$ \\
ER-MP20 & $30 \%-50 \%$ \\
C-Kit & $>90 \%$ \\
Gr-1 & $10 \%-20 \%$ \\
Mac-1 & $<1 \%$ \\
F4/80 & 0 \\
\hline
\end{tabular}

Seven-day PU.1-/- myeloid colonies were pooled and cytospun onto glass slides. Cells were fixed and stained as indicated in Materials and Methods. No background staining was observed with isotype antibody controls.

*The percentage of cells positive for the antibody tested is given. 
A

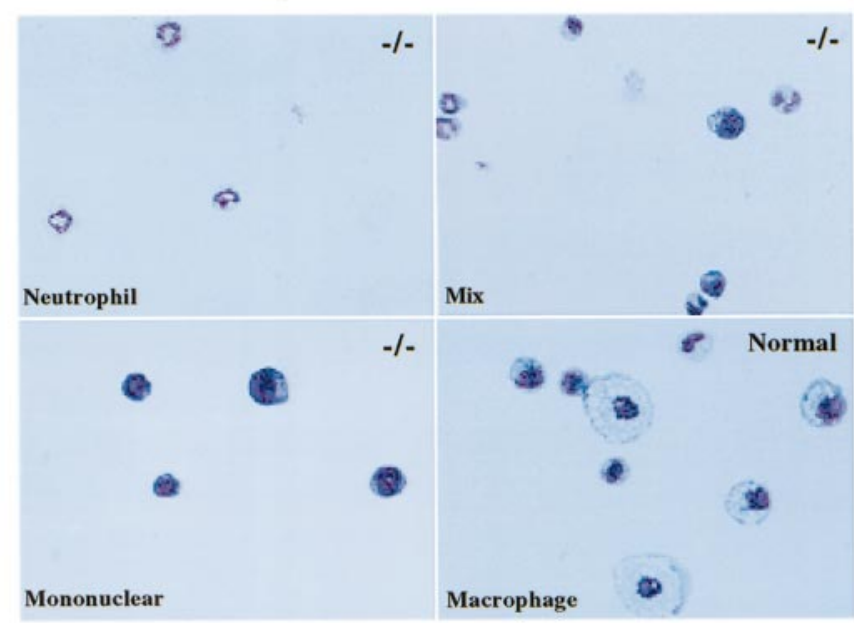

B

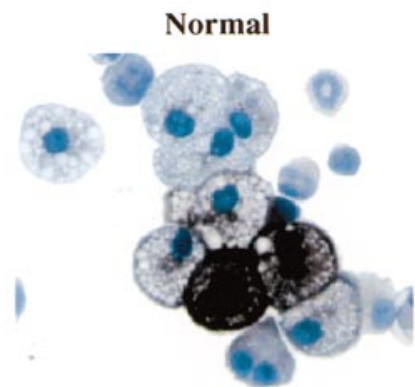

C

Normal

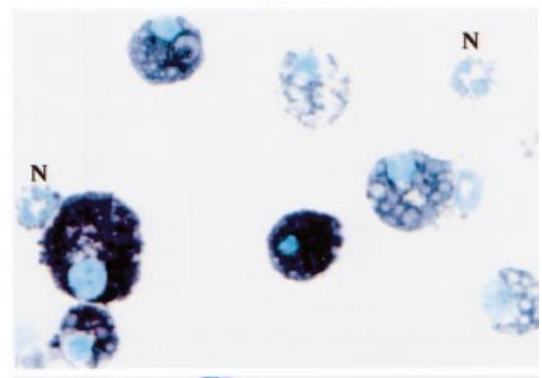

PU.1-/-

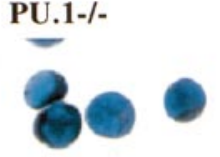

a

Moma-2

)

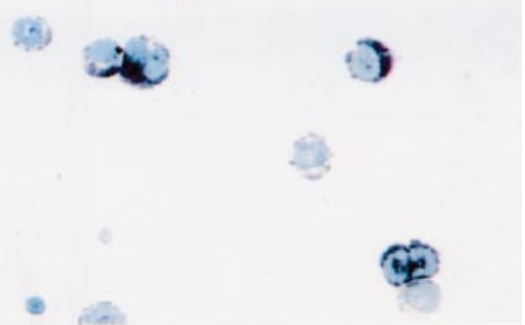

Fig 1. Characterization of CFU-derived early PU.1-/ - myeloid cells. Normal and PU.1-/ - neonate liver cells were grown in methylcellulose CFU plates for 7 days with $50 \mathrm{ng} / \mathrm{mL}$ stem cell factor (SCF), $100 \mathrm{U} / \mathrm{mL}$ IL-3, $2.5 \mathrm{ng} / \mathrm{mL} \mathrm{GM-CSF}$, and 5,000 U/mL M-CSF. (A) Individual PU.1-/myeloid colonies were harvested and stained with Wright-Giemsa stain. $-/-$ in the upper right-hand comer of the panels indicates PU.1 null colonies. A normal macrophage colony is shown in the lower right-hand panel. (B) Pooled normal or PU.1-/ - colonies were examined for NSE activity using $\alpha$-naphthyl acetate as the substrate. The dark staining cells show an NSE-positive reaction. (C) Pooled normal and PU.1-/colonies were tested for Moma-2 expression. Biotinylated secondary antibody and avidin peroxidase were used to identify Moma-2-expressing cells. Purple staining cells indicate Moma-2 expression. Moma-2 negative neutrophils (N) are indicated in the normal panel.

A

Normal

PU.1 - -

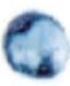

\section{Control}

B

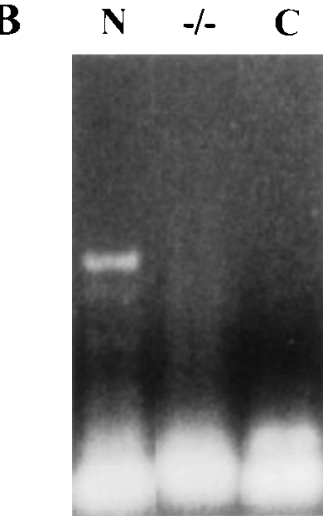

PU.1

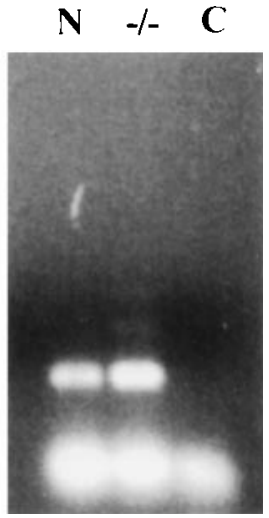

GMR

Fig 2. (A) Normal and PU.1-/ - myeloid colonies contain cells that express GM-CSF receptor $\alpha$ (GMR). A 1:10 dilution of an affinity purified rabbit polyclonal antibody to a cytoplasmic portion of GMR was used to stain pooled nomal or PU.1-/ - colonies. The same concentration of preimmune rabbit control antibody was also used. (B) The expression of GMR was verified by reverse transcriptase-PCR (RT-PCR). Normal (N) and PU.1-/ - colonies were harvested, and total RNA was extracted. Two micrograms of total RNA was used for CDNA synthesis and PCR. Expression of both GMR and PU.1 was examined. PCR conditions: $94^{\circ} \mathrm{C} 1^{\prime \prime}, 60^{\circ} \mathrm{C} 1^{\prime \prime}$, and $72^{\circ} \mathrm{C} 2^{\prime}$, for 40 cycles. A no-cDNA control (C) was included in this assay. 
contaminated the PU.1-/- CFU plates, we also looked for PU.1 message. Normal myeloid colonies expressed PU.1 message, but no message was detected in PU.1-/- colonies.

We showed that progenitors from normal neonate liver were able to form myeloid colonies in the presence of GM-CSF alone, whereas PU.1-/- progenitors did not form any colonies. ${ }^{6}$ In addition, PU.1 null cells from neonate liver were not able to proliferate in the presence of GM-CSF alone. ${ }^{6}$ Therefore, although PU.1-/- cells expressing GMR can be detected from these CFU assays with mixed growth factors, no measurable response to GM-CSF has been shown. Because the number of PU.1-/- progenitors were reduced relative to normal mice, the frequency of GM-CSF-responsive PU.1 null progenitors may be very low and might not be detected in either a CFU or proliferative assay. To maximize the input of GM-CSFresponsive progenitors, we generated single-cell suspensions of whole fetal liver from PU.1 null animals and cultured the entire mix in the presence of GM-CSF. We chose the fetal liver, because at this stage of development, this organ is the primary site of hematopoiesis and would likely be a better source of early myeloid progenitors compared with neonate liver. ${ }^{28}$ At birth, the bone marrow becomes the predominate site of hematopoiesis, while liver hematopoiesis begins to wane. ${ }^{28}$ In PU.1-/- neonates, bone marrow formation is deficient and contains a minimal number of cells. ${ }^{3}$ After 7 to 10 days, normal fetal liver cultures in the presence of GM-CSF primarily contained macrophages. In contrast to the CFU and proliferation assays, a population of early myeloid cells grew out of the PU.1 null fetal liver cultures in the presence of GM-CSF alone. These PU.1-/- early myeloid cells were found to be GM-CSFdependent, and a stable culture was established away from the fetal liver stroma and has been passaged over several months.

The generation of GM-CSF-dependent PU.1-/- cells from fetal liver cultures was repeated three times. In addition, fifteen PU.1-/- GM-CSF-dependent clones have been generated. One of the GM-CSF-dependent clones (GM-pu12798-4 or GM-pu4) was further characterized. GM-pu4 cells were analyzed by flow cytometry for the expression of various myeloid markers. These cells coexpressed markers found on early monocytic precursors, including Moma-2, ER-MP12, ERMP20, and ER-MP58 (Fig 3). As previously mentioned, monocytic precursors express Fc receptors for IgG. A monoclonal antibody that recognizes both CD16 (FcyIII) and CD32 (Fc $\gamma$ RII) positively stained GM-pu4 cells. GM-pu4 cells did not express CD11b or F4/80, but did express low levels of Gr-1. GM-pu4 cells also expressed CD18 and low levels of c-Kit but were Sca-1 negative.

Although many of the markers idenitified on the GM-pu4 cells can also be found on cells of the granulocytic lineage, the expression of Moma-2 indicated that these cells were of the monocytic lineage. To verify that GM-pu4 cells were of the monocytic lineage, they were tested for NSE activity. These cells were found to be NSE positive and sensitive to NaF treatment (Fig 4A). Another feature of early monocytic precursors is that they display phagocytic capabilities. GM-pu4 cells were incubated for 1 or 4 hours with fluorescent latex beads to determine if they were capable of phagocytosis. Cells were harvested and analyzed by flow cytometry. After 1 hour, the majority of cells had engulfed some latex beads, and by 4 hours, there were very few cells remaining that had not taken up some of the beads (Fig 4B). As a control for nonspecific sticking of the latex beads to the cells, the beads were added to the cells for 5 minutes and then washed. More than $95 \%$ of the cells were unstained (data not shown). In addition, approximately $90 \%$ of a myeloblast cell line, M1, was unstained after a 90-minute incubation with the latex beads, demonstrating that not all cells will phagocytize the latex beads (data not shown). The engulfment of the latex beads by GM-pu4 cells was verified visually using a fluorescent microscope. There was some variability in the number of particles phagocytosed by the cells, which was consistent with the broad FL-1 peak shown by the flow cytometric analysis.

These results show that the GM-CSF-dependent cells possess monocytic characteristics similar to the early PU.1-/myeloid cells characterized in the CFU assay.

Flow cytometric analysis of primary normal and PU.1 null liver cells using antibodies to monocytic precursor antigens. The data presented thus far show that under in vitro culturing conditions, PU.1-/- cells with monocytic characteristics are present. We next wanted to determine whether or not similar cells exist in vivo. A single cell suspension of normal and PU.1 null neonate liver cells was double stained with Moma-2 and either ER-MP12 or ER-MP20 monoclonal antibodies. After staining, the liver cells were analyzed by flow cytometry (Fig 5A). Normal liver showed both ER-MP12 and ER-MP20 single positive populations. In contrast, the PU.1-/- liver contained few single positive ER-MP12 or ER-MP20 cells. The scarcity of ER-MP12 and ER-MP20 single positive cells is consistent with the published data showing that the PU.1 null mice are deficient in lymphocytes and granulocytes. ${ }^{1,2}$ On the other hand, both normal and PU.1-/- livers did contain a comparable proportion of ER-MP12 ${ }^{+}$Moma- $2^{+}$and ER-MP20 ${ }^{+}$Moma- $^{+}$ double positive cells.

Further characterization indicated that the population of normal and PU.1-/- double positive cells was different. In these experiments, normal and PU.1 null liver cells were triple stained with Moma-2 and ER-MP12, along with either F4/80, CD11b, or Gr-1 antibodies (Fig 5B). Most of the cells in the normal ER-MP12 ${ }^{+}$Moma- $2^{+}$populations were positive for the monocyte-specific marker F4/80, and the myeloid markers CD11b and Gr-1. F4/80, CD11b, and Gr-1 staining was broad from mostly low to intermediate, whereas a few cells expressed high levels of CD11b or Gr-1. The coexpression of Gr-1 with F4/80 in the ER-MP12 ${ }^{+}$Moma- $2^{+}$population is consistent with Gr-1 detection on early monocytes. ${ }^{26}$ The Gr-1 bright neutrophils were located in the ER-MP20 single positive population.

Less than $30 \%$ of the ER-MP12 $2^{+}$Moma- $2^{+}$population were found to be F4/80, CD11b, or Gr-1 negative, using a gate to subtract out cells expressing these late markers. Therefore, the double positive population consists of monocytic precursors at various stages of development. Similar results were obtained when we analyzed the normal ER-MP $20^{+}$Moma- $2^{+}$population (data not shown). In contrast, no significant F4/80 or Gr-1 expression was detected in the PU.1-/- ER-MP12 ${ }^{+}$Moma- $2^{+}$ population, although a small shift in the Gr-1 histogram plot relative to background levels indicates that some cells express low levels of this antigen. In contrast to our in vitro results, a small percentage of PU.1-/- cells in this population did 

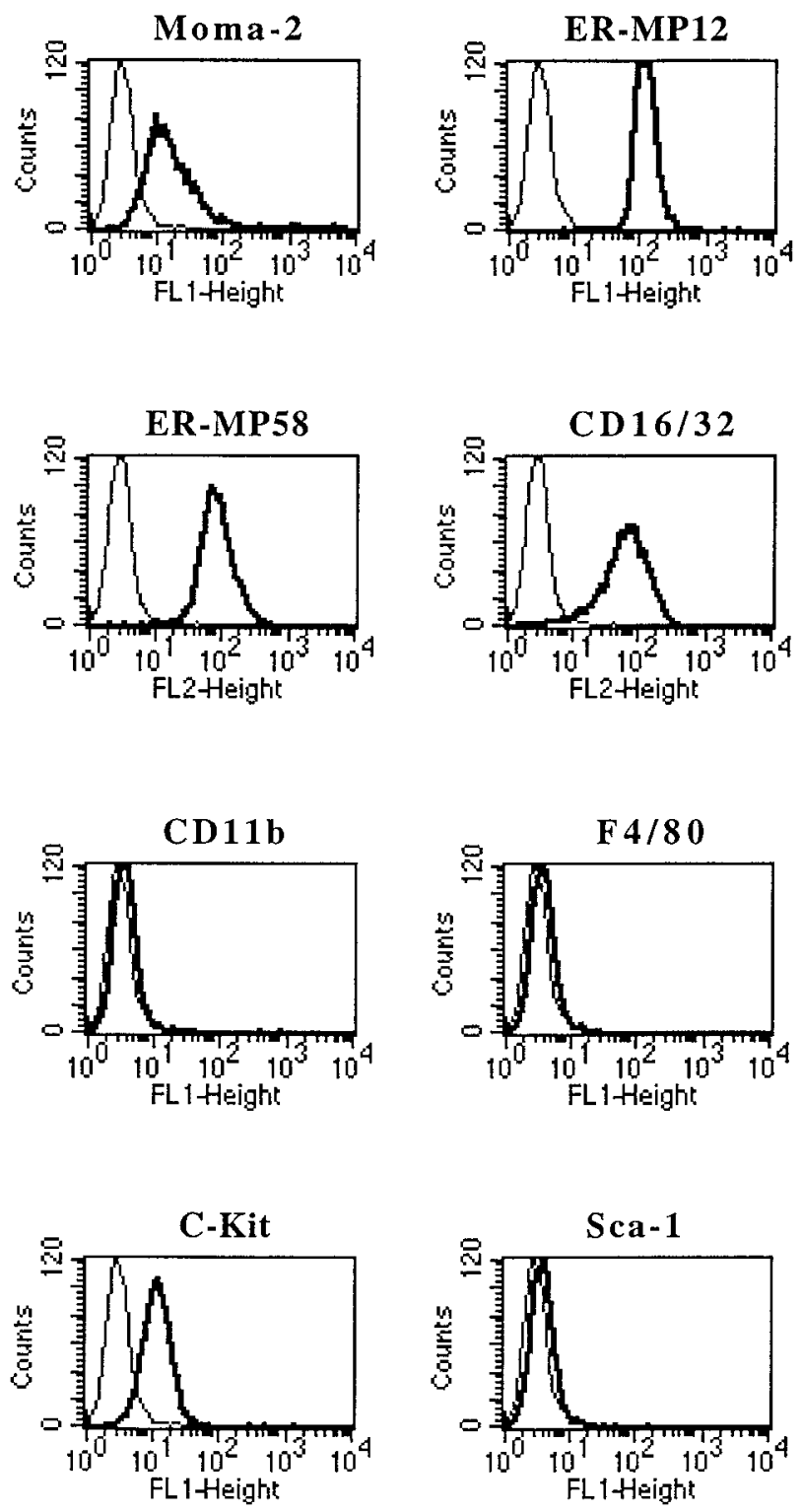

express low to intermediate levels of CD11b. Similar results were found using day 17 to 18 fetal liver from both normal and PU.1-/- animals.

To determine if the PU.1-/- double staining population contained cells with monocytic characteristics, we used antibodies with magnetic beads to isolate the ER-MP12+Moma- $2^{+}$ population from both normal and PU.1 null livers (see Materials and Methods). The sorted cells were examined for NSE activity. As shown in Fig 6 (see page 2855), both normal and PU.1-/ER-MP12 ${ }^{+}$Moma- $2^{+}$populations included cells with NSE activity and were $\mathrm{NaF}$-sensitive. It has been reported that monocytic precursors are also positive for peroxidase activity. Therefore, both the normal and PU.1-/- double staining populations were analyzed for peroxidase activity and found to contain peroxidase-positive cells (Fig 6).

The combined data from the flow cytometric and cytochemical analysis shows that a low percentage of monocytic precursors are produced in the PU.1 null mice.
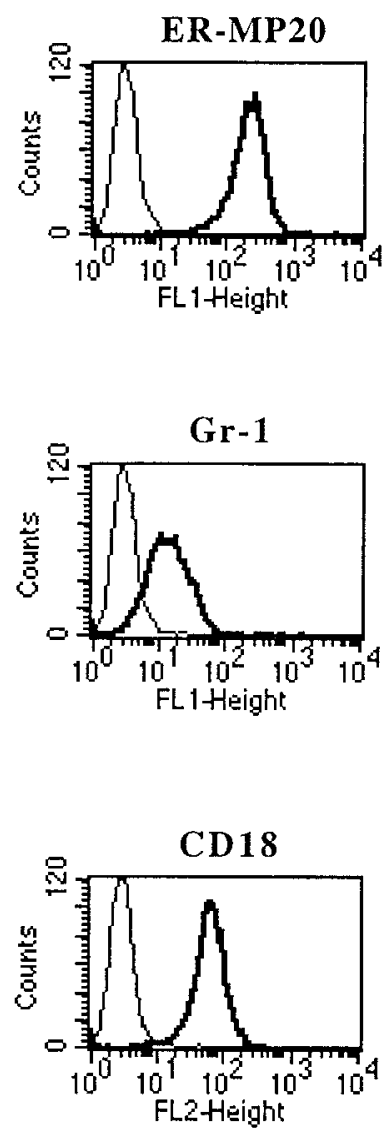

Fig 3. Antigen marker analysis of a GM-dependent PU.1-/myeloid cell line. PU.1-/ - fetal liver cultures were set up with GM-CSF. GM-dependent myeloid cells that grew from these cultures were cloned. One of the clones, GM-pu4, was analyzed by flow cytometry for the expression of various markers. Cells were incubated with either unconjugated (ER-MP12, ER-MP20, and ER-MP58), FITC-conjugated (Moma-2, Gr-1, CD11b, F4/80, cKit, and Sca-1), or PE-conjugated (CD18 and CD16/32) antibodies. FITC- or PE-conjugated secondary antibody was used to detect ER-MP12, ER-MP20, or ER-MP58. The light tracings on the histogram plots show background staining using isotype control antibodies.

\section{DISCUSSION}

Earlier reports have repeatedly shown that mature macrophages are not produced in the absence of PU.1. However, it had not been shown where in the process of monocyte/ macrophage differentiation PU.1 was required. In this report, we show that commitment to the monocytic lineage takes place without PU.1, but development beyond the stage of early monocytic precursors is blocked. Several criteria were used to identify PU.1-/- cells committed to the monocytic lineage, including morphology, expression of Moma-2, ER-MP12, ERMP20, and ER-MP58, the presence of peroxidase, NaFsensitive NSE activity and phagocytic function.

Initially, the existence of PU.1-/- monocytic precursors was shown under in vitro culturing conditions in either CFU assays or fetal liver cultures. These in vitro results were confirmed by demonstrating the presence of primary NSE and peroxidase-positive cells expressing Moma-2 and ER-MP12 in 
A

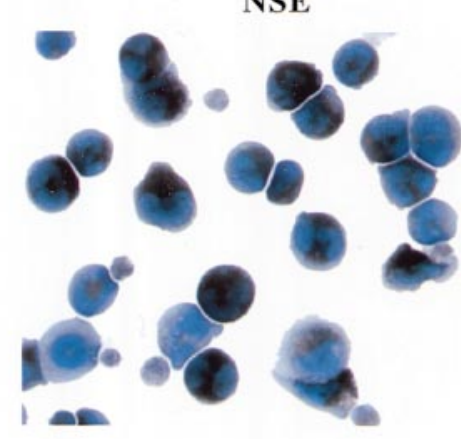

B

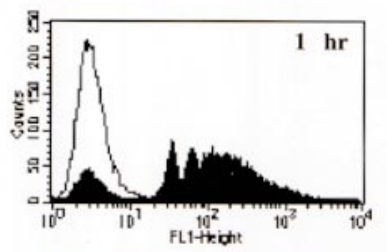
NaF to inhibit the reaction. (B) GM-pu4 cells have phagocytic activity. GM-pu4 cells were incubated with latex beads for 1 or 4 hours. The engulfiment of beads was evaluated by flow cytometry and with a fluorescent microscope. The clear tracing on the histogram plot are cells that did not receive beads. For visualization, cells were spun onto slides and stained with Wright-Giemsa. The figure shows the same two cells viewed with bright field or UV.

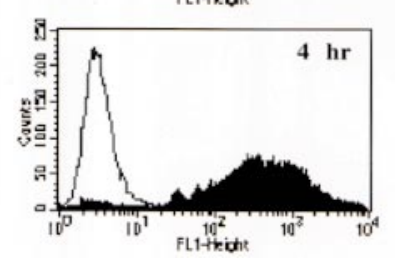

NSE

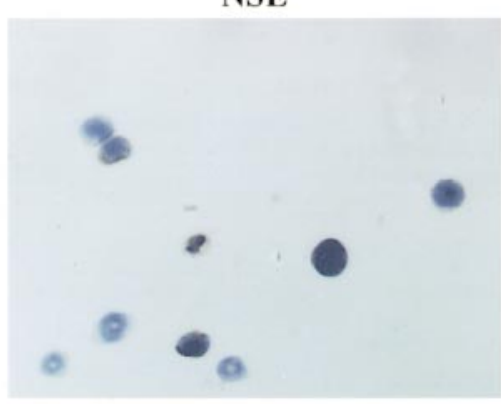

NSE

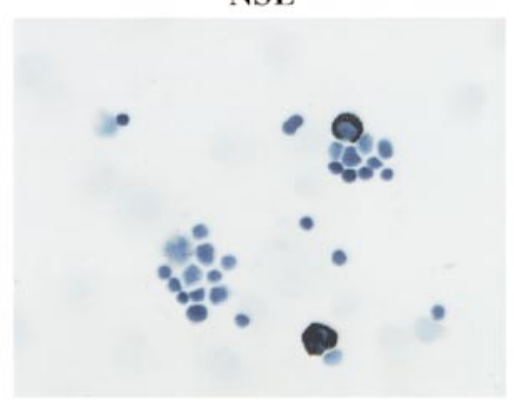

PO

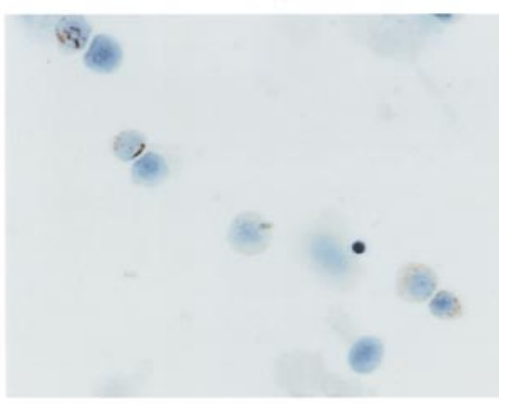

Moma-2

Fig 6. Cells showing monocytic enzyme characteristics are found in both the nomal and PU.1-/- double staining populations. The MP12 ${ }^{+}$Moma-2 ${ }^{+}$population from normal and PU.1-/ - livers was isolated using magnetically labeled antibodies (see Materials and Methods). Flow cytometry data show the enrichment of the double positive cells from (A) nomal and (B) PU.1-/ - livers. Cytochemical stains of normal or PU.1-/ - MP12+ Moma-2+ cells for NSE (black stain) and peroxidase (PO; red stain) activity are also presented. 
A

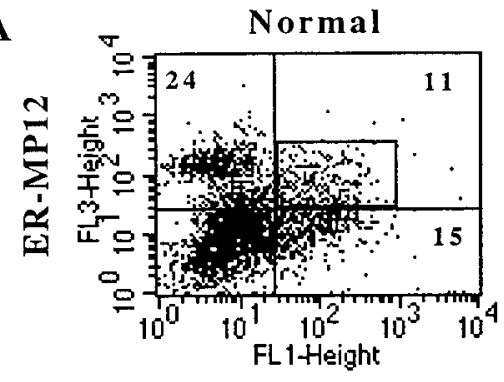

प

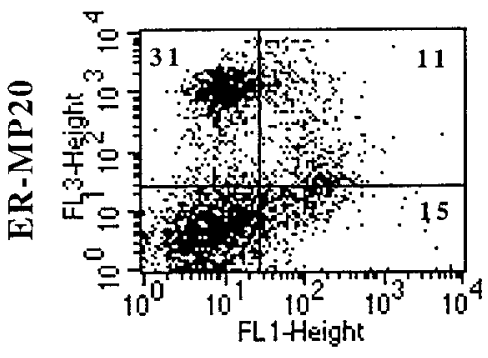

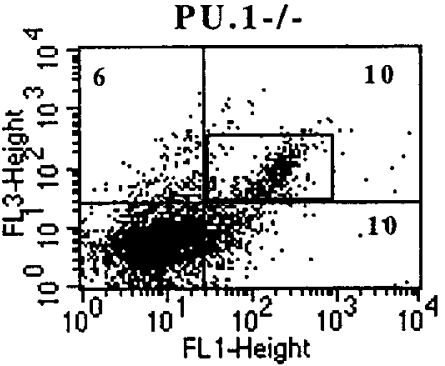

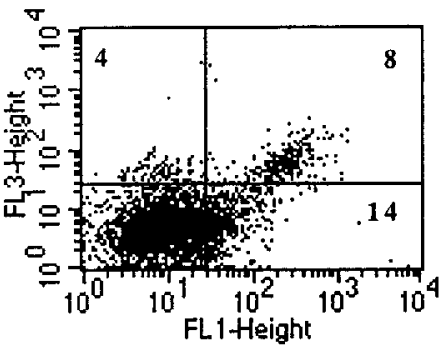

Moma-2
B

Normal

ER-MP12 ${ }^{+}$Moma-2 ${ }^{+}$
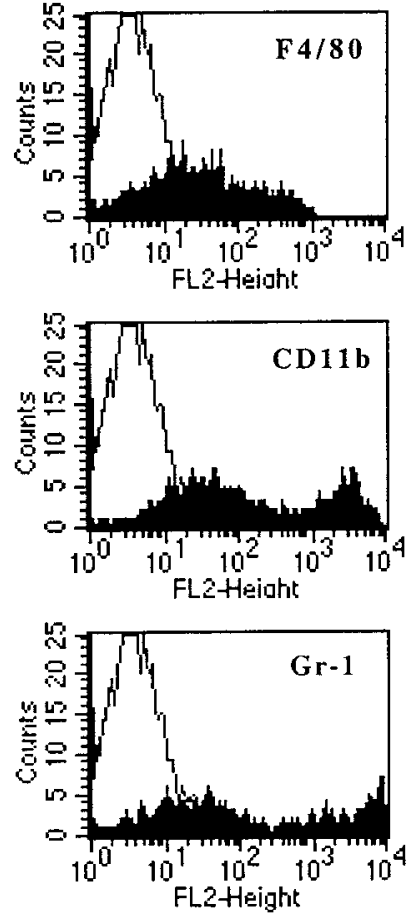

PU.1-/-

ER-MP12+Moma-2+
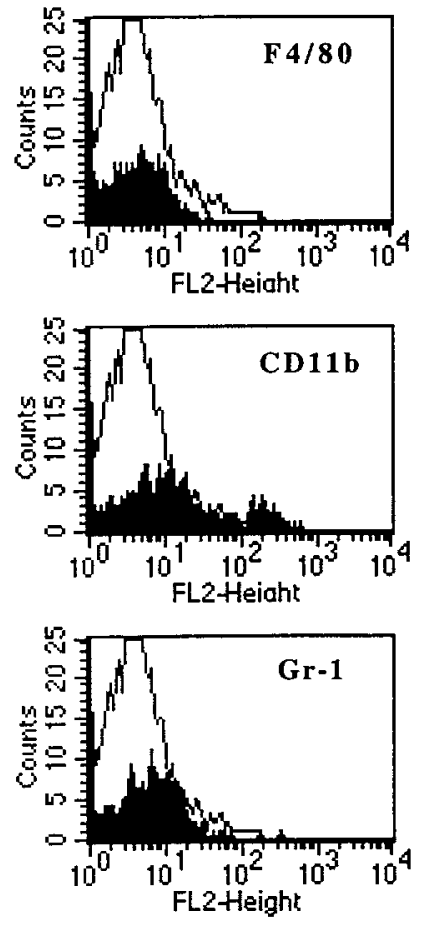

Fig 5. Monocytic precursors are found in the PU.1 null liver. Cells from normal and PU.1-/- neonate livers were triple stained with Moma-2 $2^{\mathrm{FTC}}$ and either unconjugated ER-MP12 (MP12) or ER-MP20 (MP20) monoclonal antibodies, along with F4/80 conjugated antibodies. MP12 or MP20 were stained with a biotinylated isotype-specific (IgG2a) secondary antibody followed by Streptavidin Cy-Chrome (A) Moma-2 versus MP12 or MP20 FACS data of nomal and PU.1-/- liver cells demonstrate single positive, double positive, and unstained cells. The quadrants were determined based on background staining of cells with isotype control antibodies. The percentage of each positive staining population is indicated in the comers of the quadrants. (B) Histogram plots of F4/80, Mac-1, or Gr-1 cells found in gated normal and PU.1-I- MP12+ Moma-2+ population. The rectangular box in (A) was the gate used to analyze the double positive populations. 
PU.1 null liver. Likewise, we showed the presence of cells coexpressing these early markers and later markers, including $\mathrm{CD} 11 \mathrm{~b}$ and/or low Gr-1. It has been noted that the early markers ER-MP12 and ER-MP20 can be found on cells of other hematopoietic lineages. ${ }^{14,15}$ However, in agreement with what has been reported for Moma-2, ${ }^{18}$ the coexpression of this marker with ER-MP12 and ER-MP20 can be used to specifically identify cells of the monocyte lineage. This was supported by the triple color flow cytometric analysis, which showed that the bulk of the cells in the normal double positive populations expressed late monocytic markers F4/80 and CD11b, as well as variable levels of Gr-1.

The expression pattern of ER-MP12 and ER-MP20 has been shown to delineate stages of monocyte maturation. ${ }^{16}$ Cells expressing high levels of ER-MP12 alone were defined as early monocyte precursors, including GM and macrophage (M) progenitors. As monocyte precursors matured, ER-MP12 expression decreased, and ER-MP20 expression increased. By the monocyte stage of development, cells expressed ER-MP20 but not ER-MP12. High ER-MP58 expression was also found at the early GM and M stage of development and remained constant until cells mature to macrophages, at which time the expression diminished. ${ }^{17}$ The late monocytic marker CD11b was first detected on $40 \%$ of the cells found in the population expressing both ER-MP12 and ER-MP20, and on all cells in the ER-MP20only (monocytes) population. ${ }^{16}$ Therefore, an early stage of monocyte precursors before late marker expression does occur. Consistent with these results, our analysis of the primary normal double positive population from neonate liver did show a small percentage of cells that expressed only Moma-2 with ER-MP12 and/or ER-MP20. We would argue that the early PU.1-/monocytic precursors detected in vitro and in vivo represent normal stages in mononuclear phagocyte development. The majority of PU.1-/- monocytic precursors detected were at the pre-late marker stage of development, expressing only Moma-2, ER-MP12, ER-MP58, and ER-MP20. However, some PU.1-/- monocytic precursors did mature to the late marker stage and expressed CD11b and/or low levels of Gr-1. The ability of PU.1-/- monocytic precursors to mature to the late marker stage could also account for the detection of rare F4/80 positive cells in older PU.1 null animals. ${ }^{2}$

The lack of complete macrophage development in the PU.1 null animal may be caused by an inability to respond to cytokines known to regulate monocyte/macrophage development. Cytokines such as M-CSF, GM-CSF, and IL-3 have been shown to regulate macrophage development. ${ }^{27}$ Although no M-CSF response has been shown in cells from the PU.1-/mouse, PU.1-/- progenitors have been shown to respond to $\mathrm{IL}^{3}{ }^{6}$ and now to GM-CSF. Not only do the PU.1-/progenitors respond to GM-CSF, but the cells that grow out from the PU.1-/- fetal liver culture were characterized as belonging to the monocytic lineage. Interestingly, GM-CSFdependent cells could not be derived from PU.1-/- neonate liver cultures (data not shown). It is unclear as to why there is a change in response to GM-CSF from PU.1-/- fetal liver cultures compared with neonate liver cultures. As already mentioned, one explanation could be that the number of progenitors in the liver expressing GMR is extremely low at birth in the PU.1-/- animal. An alternative explanation, although not mutually exclusive, may be that the stroma in the fetal liver cultures provide additional secreted or surface-bound factors that prime PU.1 null progenitors to respond to GM-CSF and proliferate. In support of this theory, a study was published in which the investigators found that adult bone marrow and fetal liver stroma provided a better environment to maintain GM-CFC compared with neonate liver. ${ }^{29}$ This is consistent with both the adult bone marrow and fetal liver being active sites of hematopoiesis, whereas neonate liver has low hematopoietic activity. ${ }^{28}$

It is possible that the inability of IL-3 and GM-CSF to enable complete macrophage maturation in the PU.1 null mice is that PU.1 is required to receive signals initiated by these cytokines through their receptors. After receiving IL-3- or GM-CSF-induced signals, PU.1 may modulate the expression of genes required for monocyte/ macrophage development. We have shown that PU.1 is important for mediating M-CSF- and GM-CSF-induced proliferation of bone marrow-derived macrophages. ${ }^{30}$ However, it is clear from studies on mice lacking M-CSF or both M-CSF and GM-CSF, that macrophage development proceeds in the absence of these growth factors, although in some tissues the level of production is diminished. ${ }^{31,32}$ Similarly, mice deficient in both the common $\beta c$ chain used by the IL-3 and GM-CSF receptor and the IL-3 ligand were able to generate monocytes. ${ }^{33}$ Therefore, PU.1 likely regulates monocyte/macrophage development independent of growth factor receptor-mediated signaling.

\section{ACKNOWLEDGMENT}

The authors thank Dr David Hume for the IL-3 and M-CSF. They also thank Drs Bruce Torbett, Karen Anderson, and Melody Clark for their critical review of this manuscript.

\section{REFERENCES}

1. Scott EW, Simon MC, Anastasi J, Singh H: Requirement of transcription factor PU.1 in the development of multiple hematopoietic lineages. Science 265:1573, 1994

2. McKercher SR, Torbett BE, Anderson KL, Henkel GW, Vestal DJ, Baribault H, Klemsz M, Feeney AJ, Wu GE, Paige CJ, Maki RA: Targeted disruption of the PU.1 gene results in multiple hematopoietic abnormalities. EMBO J 15:5647, 1996

3. Tondravi MM, McKercher SR, Anderson K, Erdmann JM, Quiroz M, Maki R, Teitelbaum SL: Osteopetrosis in mice lacking haematopoietic transcription factor PU.1. Nature 386:81, 1997

4. Olson MC, Scott EW, Hack AA, Su GH, Tenen DG, Singh H, Simon MC: PU.1 is not essential for early myeloid gene expression but is required for terminal myeloid differentiation. Immunity 3:703, 1995

5. Scott EW, Fisher RC, Olson MC, Kehrli EW, Simon MC, Singh H: PU.1 functions in a cell-autonomous manner to control the differentiation of multipotential lymphoid-myeloid progenitors. Immunity 6:437, 1997

6. Anderson KL, Smith KA, Conners K, McKercher SR, Maki RA, Torbett BE: Myeloid development is selectively disrupted in PU.1 null mice. Blood 91:3702, 1998

7. DeKoter RP, Walsh JC, Singh H: PU.1 regulates both cytokinedependent proliferation and differentiation of granulocyte/macrophage progenitors. EMBO J 17:4456, 1998

8. Henkel GW, McKercher SR, Yamamoto H, Anderson KL, Oshima RG, Maki RA: PU.1 but not Ets-2 is essential for macrophage development from embryonic stem cells. Blood 88:2917, 1996

9. van Furth R: Origin and turnover of monocytes and macrophages. Curr Top Pathol 79:125, 1989

10. Hirsch S, Austyn JM, Gordon S: Expression of the macrophage- 
specific antigen F4/80 during differentiation of mouse bone marrow cells in culture. J Exp Med 154:713, 1981

11. Morioka Y, Naito M, Sato T, Takahashi K: Immunophenotypic and ultrastructural heterogeneity of macrophage differentiation in bone marrow and fetal hematopoiesis of mouse in vitro and in vivo. J Leukoc Biol 55:642, 1994

12. van Furth R, Hirsch JG, Fedorko ME: Morphology and peroxidase cytochemistry of mouse promonocytes, monocytes and macrophages. J Exp Med 132:794, 1970

13. Goud TJLM, Schotte C, van Furth R: Identification and characterization of the monoblast in mononuclear phagocyte colonies grown in vitro. J Exp Med 142:1180, 1975

14. Leenen PJM, Melis M, Slieker WAT, Van Ewijk W: Murine macrophage precursor characterization II. Monoclonal antibodies against macrophage precursor antigens. Eur J Immunol 20:27, 1990

15. Leenen PJM, de Bruijn MFTR, Voerman JSA, Campbell PA, van Ewijk W: Markers of mouse macrophage development detected by monoclonal antibodies. J Immunol Methods 174:5, 1994

16. de Bruijn MFTR, Slieker WAT, van der Loo JCM, Voerman JSA, van Ewijk W, Leenen PJM: Distinct mouse bone marrow macrophage precursors identified by differential expression of ER-MP12 and ERMP20 antigens. Eur J Immunol 24:2279, 1994

17. de Bruijn MFTR, Ploemacher RE, Mayen AEM, Voerman JSA, Slicker WAT, van Ewijk W, Leenen PJM: High-level expression of the ER-MP58 antigen on mouse bone marrow hematopoietic progenitor cells marks commitment to the myeloid lineage. Eur J Immunol 26:2850, 1996

18. Kraal G, Rep M, Janse M: Macrophages in T and B Cell compartments and other tissue macrophages recognized by monoclonal antibody Moma-2. Scand J Immunol 26:653, 1987

19. Slieker WAT, de Rijk-de Bruijn MFTR, Leenen PJM, van Ewijk W: ERMP12 antigen, a new cell surface marker on mouse bone marrow cells with thymus-repopulating ability: I. Intrathymic repopulating ability of ER-MP12-positive bone marrow cells. Int Immunol 5:1093, 1993

20. van der Loo JCM, Slieker WAT, Ploemacher RE: Use of ER-MP12 as a positive marker for the isolation of murine long-term in vitro repopulating stem cells. Exp Hematol 23:1002, 1995

21. Ling V, Luxenberg D, Wang J, Nickbarg E, Leenen PJ, Neben S, Kobayashi M: Structural identification of the hematopoietic progenitor antigen ER-MP12 as the vascular endothelial adhesion molecule PECAM-1 (CD31). Eur J Immunol 27:509, 1997

22. McCormack JM, Leenen PJM, Walker WS: Macrophage progenitors from mouse bone marrow and spleen differ in their expression of the Ly-6c differentiation antigen. J Immunol 151:6389, 1993

23. Jain N: Schalm's Veterinary Hematology. Philadelphia, PA, Lea \& Febiger, 1986

24. Yam LT, Li CY, Crosby WH: Cytochemical identification of monocytes and granulocytes. Am J Clin Pathol 55:283, 1971

25. Li CY, Lam KW, Yam LT: Esterases in human leukocytes. J Histochem Cytochem 21:1, 1973

26. Hestdal K, Ruscetti FW, Ihle JN, Jacobsen SEW, Dubois CM, Kopp WC, Longo DL, Keller JR: Characterization and regulation of RB6-8C5 antigen expression on murine bone marrow cells. J Immunol 147:22, 1991

27. Quesenberry PJ: Hemopoietic stem cells, progenitor cells, and cytokines, in Williams W, Beutler E, Erslev A, Lichtman M (eds): Hematology. New York, NY, McGraw-Hill, 1990, p 211

28. Morrison SJ, Uchida N, Weissman IL: The biology of hematopoietic stem cells. Ann Rev Cell Dev Biol 11:35, 1995

29. van Den Heuvel R, Schoeters G, Leppens H, Vanderborght O: Stromal cells in long-term cultures of liver, spleen, and bone marrow at different developmental ages have different capacities to maintain GM-CFC proliferation. Exp Hematol 19:115, 1991

30. Celada A, Borras FE, Soler C, Lloberas J, Klemsz M, van Beveren C, McKercher S, Maki RA: The transcription factor PU.1 is involved in macrophage proliferation. J Exp Med 184:61, 1996

31. Begg SK, Radley JM, Pollard JW, Chisolm OT, Stanley ER, Bertoncello I: Delayed hematopoietic development in osteopetrotic (op/op) mice. J Exp Med 177:237, 1993

32. Nilsson SK, Lieschke GJ, Garcia-Wijnen CC, Williams B, Tzelepis D, Hodgson G, Grail D, Dunn AR, Bertoncello I: Granulocyte-macrophage colony-stimulating factor is not responsible for the correction of hematopoietic deficiencies in the maturing op/op mouse. Blood 86:66, 1995

33. Nishinakamura R, Miyajima A, Mee PJ, Tybulewicz VL, Murrary R: Hematopoiesis in mice lacking the entire granulocytemacrophage colony-stimulating factor/interleukin-3/interleukin-5 functions. Blood 88:2458, 1996 